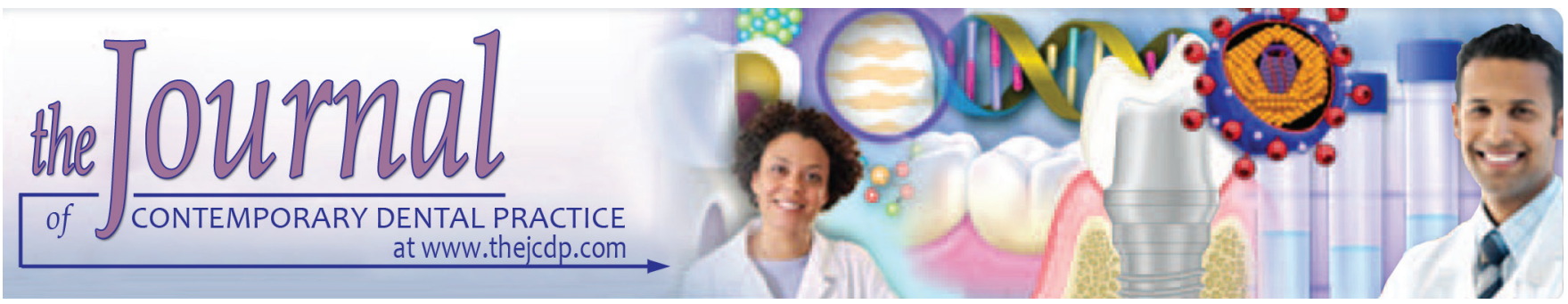

\title{
Intricate Assessment and Evaluation of Dental Implants in Patients on Bisphosphonate Therapy: A Retrospective Analysis
}

${ }^{1}$ Suraj Suvarna, ${ }^{2}$ Poonam Dutt, ${ }^{3}$ Animesh Misra, ${ }^{4}$ Nausheen Usmani, ${ }^{5}$ Abhishek Singh, ${ }^{6}$ Chandini Suvarna

\section{ABSTRACT}

Background: Osteonecrosis is one of the prevalent side effects of bisphosphonate (BP) therapy in oral cancer patients. In case of patients with various oncologic lesions, standard guidelines contraindicate the use of dental implants if the patients are on BP therapy. Literature also quotes studies that emphasize on the safety of dental implants in patients on BP therapy. Hence, this study aimed to retrospectively evaluate the clinical outcome of dental implants in patients on BP therapy.

Materials and methods: In this retrospective analysis, a total of 140 dental implants in 112 patients were included. Inclusion criteria included only those patients who were on or had history of $\mathrm{BP}$ therapy. Calculation of implant failure and survival rate was done.

Results: Ten cases of implant failure occurred, giving a success rate of above $92 \%$, which was comparable to the results found in previous studies in patients who were not on BP therapy.

Conclusion: No significant risk of implant failure is seen in patients on BP therapy compared with other patients.

Keywords: Bisphosphonate, Implants, Osteonecrosis of the jaws.

How to cite this article: Suvarna S, Dutt P, Misra A, Usmani N, Singh A, Suvarna C. Intricate Assessment and Evaluation of Dental Implants in Patients on Bisphosphonate Therapy: A Retrospective Analysis. J Contemp Dent Pract 2016;17(5):414-417.

Source of support: Nil

Conflict of interest: None

1,3,4 Department of Prosthodontics, Shree Bankey Bihari Dental College and Research Centre, Ghaziabad, Uttar Pradesh, India

${ }^{2}$ Department of Periodontics, Shree Bankey Bihari Dental College and Research Centre, Ghaziabad, Uttar Pradesh, India

${ }^{5}$ Department of Prosthodontics, Sudha Rustagi College of Dental Sciences and Research, Faridabad, Haryana, India

${ }^{6}$ Private Practice, New Delhi, India

Corresponding Author: Suraj Suvarna, Professor, Department of Prosthodontics, Shree Bankey Bihari Dental College and Research Centre, Ghaziabad, Uttar Pradesh, India, e-mail: drsuraj@hotmail.com

\section{INTRODUCTION}

One of the much prevalent side effects associated with bisphosphonate (BS) therapy in patients with malignant disorders is osteonecrosis of the jaws (ONJ), with a prevalence rate as high as $20 \%$ in those patients. ${ }^{1,2}$ Bisphosphonates comprise bone resorption inhibiting drugs that inhibit chemotaxis by reducing the lifespan of osteoclasts and causing their apoptosis. Whereas oral BS plays an important role in treating osteoporosis, Paget's disease, and imperfect osteogenesis, BS administered intravenously causes reduction in pain, hypercalcemia in malignant conditions, Paget's disease-induced skeletal complications, and also in treating cancer-associated skeletal complications. ${ }^{3,4}$

The first case of ONJ in cancer patients on BP therapy was reported by Wang et al along with BP therapy; these patients were also on other medications, including chemotherapy drugs, corticosteroids, etc. ${ }^{5}$ It is only after invasive dental treatment that the cases of BP-associated ONJ are diagnosed. Spontaneous occurrence of BP-associated ONJ occurs very less commonly. ${ }^{6}$ Metastatic breast cancers and multiple myeloma were the most common malignancies in which $\mathrm{BP}$-associated ONJ was known to occur initially. ${ }^{7}$ Also, it is seen that $\mathrm{ONJ}$ occurred in much higher frequency in malignant cases compared with benign cases. ${ }^{8}$

Guidelines regarding the use of BP therapy for the treatment in patients with oncologic pathologies contraindicate the use of implant therapy. Conversely, literature quotes studies that emphasize the dental implant safety in patients on BP therapy. Review of the literature highlights very less retrospective analyses on this topic. As far as dental implant surgeries are concerned, $\mathrm{BP}$ therapy is not in the list of absolute contraindication. 
Furthermore, risk assessment of the patients should be done in which dental implants are required for rehabilitation and prior information should be given to the patients regarding the potential consequences of BP therapy. ${ }^{9}$ Keeping all these facts in mind, the authors have genuinely attempted to retrospectively evaluate the overall clinical outcome of dental implants in patients on BP therapy.

\section{MATERIALS AND METHODS}

A total of 112 cases were reviewed in this retrospective analysis. Medical records of all the patients were examined from 1995 to 2010 and only those patients that received BP therapy in any form prior to dental implant surgery were included in the study. A total of 140 dental implants were placed in 112 patients. Bone grafting was done in 82 occasions in 55 patients. Out of all the grafting procedures done, 51 were socket grafts, 8 were sinus lifting procedures, 7 closed sinus lifts, 15 guided tissue regenerations, and 1 buccal contour grafts. Alendronate was taken up by 40 patients, while 10 and 8 patients took risedronate and ibandronate respectively. Complete detailed history of the patients was taken regarding their BP therapy and thorough periodontal examination was done to check and record pocket depth and bleeding on probing. To rule out bone resorption and bone loss, periodic periapical and panoramic X-rays were taken. Detailed data were gathered from patient's records regarding description of medical problems, surgical procedures performed, medicaments taken, type of grafts used for grafting, and implant stability at the time of surgery. Only those patients for whom follow-up record of a minimum 3 years was available were included in this study. Implant failure and survival rates were calculated.

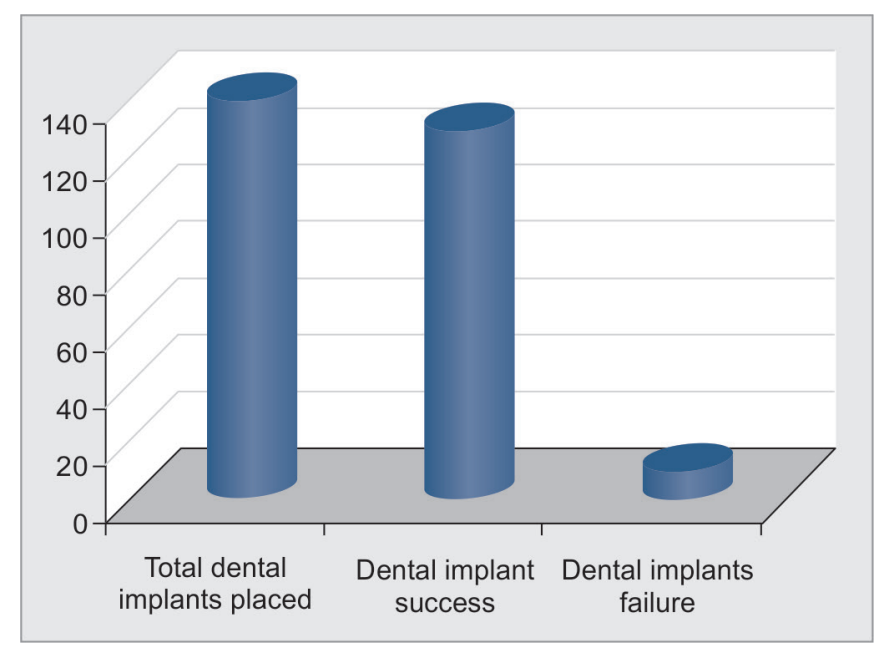

Graph 1: Total successful dental implants out of total implant surgeries placed

\section{RESULTS}

The overall dental implant failure was only $7.1 \%$ (Table 1 and Graph 1), which was almost similar to the result of dental implant success rate in patients who were not on BP therapy. ${ }^{10}$ In these 112 cases, 82 were females and 30 were males (Graph 2). Out of all the implant cases which failed, eight were seen in females and two were seen in males (Graph 3). In two implant failure cases (females), patient received multiple dental implants out of which only one failed in each case. In the remaining eight cases of dental implant failure, single implant was placed. In the two males in whom implant failure occurred, the patients were on BP therapy for 5 months, had no smoking habit, and did not undergo any kind of bone grafting procedure (Graph 4). The implant failure occurred 2 months after the implant surgery. In another three cases in which dental implant failure occurred, the patients were smokers, had a history of smoking 2 to 3 cigarettes per day for the last 2 years, and underwent bone grafting procedure during the implant placement. The implant failure occurred within 1 month of implant surgery. Two of the dental implant failure cases had a history of BP therapy for the last 1 year before the implant placement surgery, had a negative smoking history, and underwent a closed sinus lift procedure. The implant failed within 6 months of surgery. The last three cases of implant failure were on BP therapy for the last 3 years, had a smoking history, and received a sinus lift procedure. The implant failure occurred within 3 weeks of implant surgery. Osteonecrosis

Table 1: Total successful dental implants out of total implant surgeries placed

\begin{tabular}{lll}
\hline $\begin{array}{l}\text { Total dental implants } \\
\text { placed }\end{array}$ & $\begin{array}{l}\text { Dental implant } \\
\text { success }\end{array}$ & $\begin{array}{l}\text { Dental implant } \\
\text { failure }\end{array}$ \\
\hline 140 & $130(92.86 \%)$ & $10(7.14 \%)$ \\
\hline
\end{tabular}

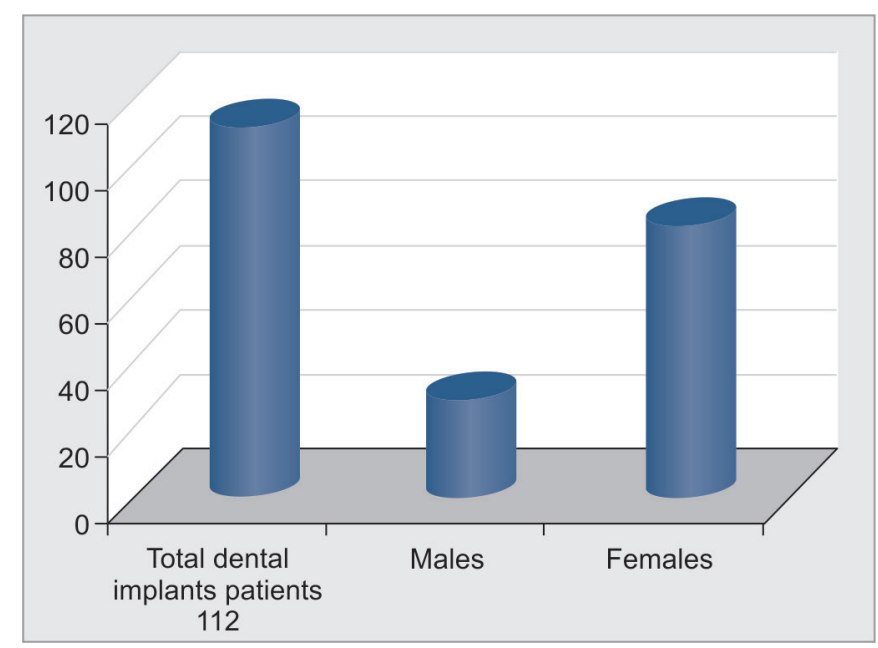

Graph 2: Distribution of total dental implant cases among males and females 


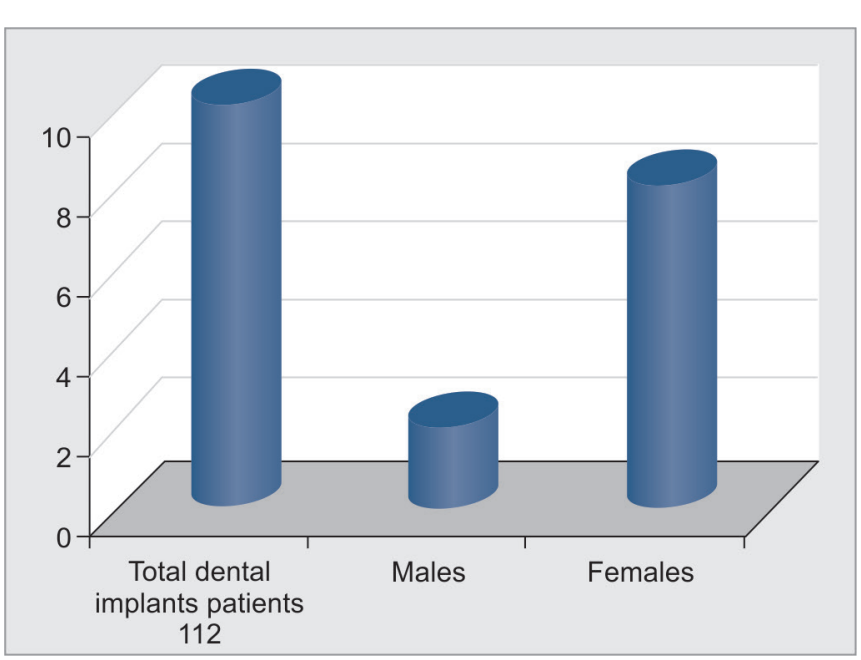

Graph 3: Distribution of dental implant failure cases among males and females

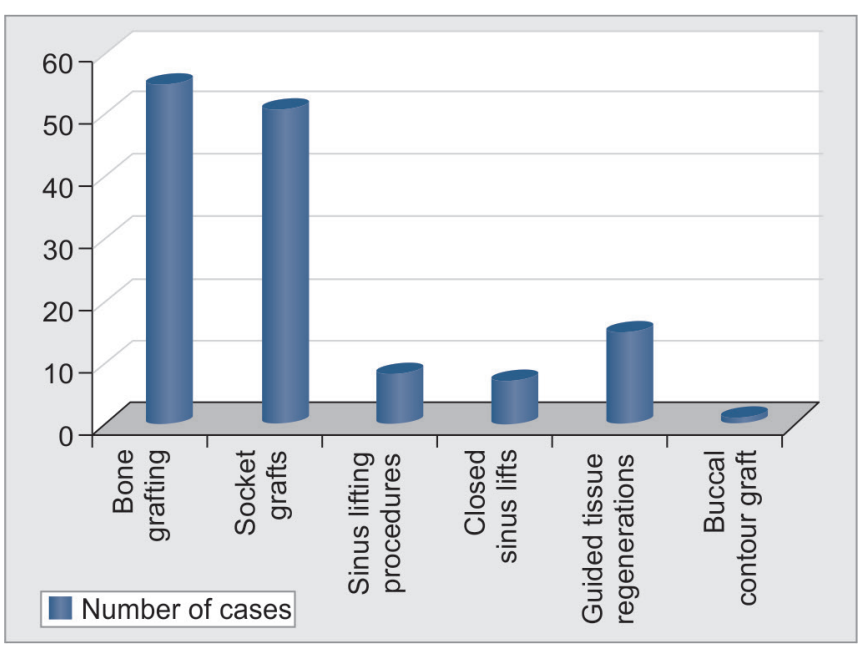

Graph 4: Distribution of dental implant cases in which additional parameters were added of the jaws was not seen in any of the patients and also none of the patients showed clinical and radiographical feature of bone loss. On 3 months follow-up, pocket depth remained unchanged and no bleeding on probing was noticed around any of the implant cases.

\section{DISCUSSION}

Under the action of parathyroid hormone (PTH), osteoclasts cause bone resorption by developing a ruffled border on the interface with the bone surface, and start secretion of hydrochloric acid. Under the action of this strong acid, demineralization of the bone matrix and organic components takes place along with increased release of bone inductive proteins [bone morphogenetic proteins (BMPs) and insulin-like growth factors], which further promote differentiation of osteoblasts and new osteoid secreti. ${ }^{11}$ When patients suffering from some kind of malignancies and bone diseases, such as cancer, Paget disease, and osteoporosis are treated with BP therapy, there is disturbance in the normal biological action of osteoclasts. There is loss of normal ruffled border of osteoclasts in response to BP reaction with osteoclasts, further causing a retraction from the bone's surface followed by its death. As a result, there is no new osteoid bone formation. ${ }^{12}$ Hence, we assessed the effect of BP therapy on the survival of dental implants.

Of the total implants, $92.1 \%$ showed success, while 10 implants failed. Among the failed implants, $70 \%$ were placed in maxillary posterior region compared with $20 \%$ of the total implants that were inserted in the maxillary area; $60 \%$ of the total implant failure occurred in patients who had smoking habit. ${ }^{10}$ These results are comparable to the results that show high failure rate of dental implants in smokers and in patients in whom implants were placed in maxillary posterior region. No causal relationship was observed in the present study between the dental implant failure rate and BP therapy.

Not even a single case of ONJ was observed in the present study along with significant high success rate of bone grafts irrespective of BP therapy. All other procedures included in this study, like sinus lift, guided tissue regeneration, had no postoperative complications. Qamheya et $\mathrm{a}^{13}$ reviewed the data available on BS-related ONJ and dental implants and highlighted that analysis of relationship between dental implants and BSs should be done, which should be multifactors: Duration, route of uptake, dosage of the drug, and patient's other medications that affect the effects of BS and therefore, all the treated patients with this drug must be informed about the risk of implant loss or possibility of osteonecrosis. Zahid et $\mathrm{al}^{14}$ retrospectively evaluated the influence of BSs on alveolar bone loss around osseointegrated implants and concluded that patients on BP might be at higher risk for implant thread exposure. Bell and Bell ${ }^{10}$ also retrospectively analyzed the effect of BP therapy on dental implants. They examined 101 dental implants in 42 patients who had taken BPs prior to surgeries involving implant placement and concluded that patients who are on oral BPs are on no higher risk of implant failure as compared with other patients. Under the light of the above results, no significant relation is seen between dental implant failure and BP therapy.

\section{CONCLUSION}

In the light of the above-mentioned results, it can be concluded that no significant risk of implant failure is seen in patients on BP therapy compared with other patients. Dental implants and its osseointegration seem to remain functionally stable in such patients. However, in patients having a history of BP therapy for $>3$ years, 
alternate treatment options and further researches are advocated to explore the detailed impact of BP therapy on success rate of dental implants.

\section{REFERENCES}

1. Grötz KA, Piesold JU, Al-Nawas B. Bisphosphonat-assoziierte Kiefernekrose (BP-ONJ) und andere Medikamentenassoziierte Kiefernekrosen; 2012. AWMF online.

2. Walter C,Al-Nawas B, Frickhofen N, Gamm H, BeckJ, Reinsch L, Christina B, Knut A, Wilfried W. Prevalence of bisphosphonate associated osteonecrosis of the jaws in multiple myeloma patients. Head Face Med 2010 Jul;6:11.

3. Marx RE, Sawatari Y, Fortin M, Broumand V. Bisphosphonateinduced exposed bone (osteonecrosis/osteopetrosis) of the jaws: Risk factors, recognition, prevention, and treatment. J Oral Maxillofac Surg 2005 Nov;63(11):1567-1575.

4. Serra MP, Llorca CS, Donat FJ. Oral implants in patients receiving bisphosphonates: A review and update. Med Oral Patol Oral Cir Bucal 2008 Dec;13(12):E755-E760.

5. Wang EP, Kaban LB, Streler GJ, Raje N, Troulis MJ. Incidence of osteonecrosis of the jaw in patients with multiple myeloma and breast or prostate cancer on intravenous bisphosphonate therapy. J Oral Maxillofac Surg 2007 Jul;65(7):1328-1331.

6. Ruggiero SL, Mehrotra B, Rosenberg TJ, Engroff SL. Osteonecrosis of the jaws associated with the use of bisphosphonates: A review of 63 cases. J Oral Maxillofac Surg 2004 May;62(5):527-534.

7. Marx RE. Pamidronate (Aredia) and zoledronate (Zometa) induced avascular necrosis of the jaws: A growing epidemic. J Oral Maxillofac Surg 2003 Sep;61(9):1115-1117.

8. Hoff AO, Toth B, Hu M, Hortobagyi GN, Gagel RF. Epidemiology and risk factors for osteonecrosis of the jaw in cancer patients. Ann NY Acad Sci 2011 Feb;1218(1):47-54.

9. Walter C, Al-Nawas B, Wolff T, Schiegnitz E, Grötz KA. Dental implants in patients treated with antiresorptive medication -A systematic literature review. Int J Implant Dent 2016 Apr;2:1-15.

10. Bell BM, Bell RE. Oral bisphosphonates and dental implants: A retrospective study. J Oral Maxillofac Surg 2008 May;66(5): 1022-1024.

11. Vijay G, Raghavan V. Bisphosphonates and osteonecrosis of jaws. J Indian Acad Oral Med Radiol 2012;24(4):330-333.

12. Robert, EM. Oral and intravenous bisphosphonate-induced osteonecrosis of the jaws. History etiology, prevention, and treatment. 2nd ed. New Malden: Quintessence Publishing Co, Inc.; 2007. p. 9-93.

13. Qamheya AHA, Yeniyol S, Arısan V. Bisphosphonate-related osteonecrosis of the jaw and dental implants. J Istanbul Univ Fac Dent 2016;50(1):59-64.

14. Zahid TM, Wang BY, Cohen RE. Influence of bisphosphonates on alveolar bone loss around osseointegrated implants. J Oral Implant 2011 Jun;37(3):335-346. 